\title{
Effects of Lattice Vibrations on Angular Correlations*
}

\author{
L. H. TANG $\dagger$ \\ Physics Department, University of Detroit, Detroit, Michigan \\ AND \\ R. K. OSBORN \\ Department of Nuclear Engineering, University of Michigan, Ann Arbor, Michigan
}

(Received 5 August 1965; revised manuscript received 31 January 1966)

\begin{abstract}
A theory of the influence of the environment on $\gamma-\gamma$ angular correlation is presented. The theory is formulated by using the damping theory, and the perturbation factor of the perturbed angular-correlation function, which contains the effects of the changes of the states of the environment on the angular correlation, is obtained. In the solid environment, the effects of the lattice vibrations on the electric quadrupole coupling have been analyzed by using the normal-mode expansion of the lattice displacements; and the perturbed angular correlation function is shown to be a function of the crystal temperature. In order to determine the nuclear electric quadrupole moment in an excited state, which cannot be done by the usual microwave methods, a rotational technique is suggested and the theory has been developed for the case of asymmetric fields. For the special case of an axially symmetric crystalline field, the present theory predicts a phase shift in the rotational pattern of anisotropy.
\end{abstract}

\section{INTRODUCTION}

$I^{1}$ $\mathrm{N}$ recent years, several experiments ${ }^{1-7}$ have pointed up the need for a generalization of the theory of environmental effects on the perturbation of $\gamma-\gamma$ angular correlations. The conventional theory has treated the environment as a static magnetic-dipole or electricquadrupole field of prescribed symmetry. However, it has been observed that the environmental perturbation of the angular correlation is often measurably temperature-dependent, indicating a significant coupling between the decaying nuclei and the dynamical characteristics of their surroundings. It is the purpose of this work to incorporate this dynamic coupling into a calculation of the angular-correlation perturbation factor. The present approach to the problem utilizes an adaptation of Heitler's damping theory ${ }^{8}$ of transitions among decaying states. Since the strictly computational details of this treatment of transition probabilities have been discussed extensively elsewhere, we give them no further consideration here. The physical model envisages the decaying nucleus as an integral part of a larger system to which its translational and orientational degrees of freedom may be more or less tightly bound, depending upon whether the large system is a solid,

* Supported in part by the U. S. National Science Foundation $\dagger$ This paper is based on a part of the dissertation submitted by L. H. Tang in partial fulfillment of the requirements for the Ph.D. degree in Nuclear Science, Nuclear Engineering Department, The University of Michigan.

${ }^{1}$ P. J. Ouseph and F. L. Canavan, Phys. Letters 3, 143 (1962).

${ }^{2}$ H. Albers-Schönberg et al., Phys. Rev. 90, 322 (1953).

${ }^{3} \mathrm{H}$. Albers-Schönberg et al., Phys. Rev. 91, 1287 (1953).

${ }^{4} \mathrm{H}$. Albers-Schönberg et al., Helv. Phys. Acta 27, 547 (1954).

${ }^{5}$ R. R. Hewitt and T. T. Taylor, Phys. Rev. 125, 524 (1962).

${ }^{6} \mathrm{M}$. Salomon et al., in Perturbed Angular Correlation, edited by E. Karlsson et al. (North-Holland Publishing Company, Amsterdam, 1964), p. 273.

${ }^{7}$ R. W. Sommerfeldt and L. Schecter, Phys. Letters 3, 5 $(1962 / 63)$.

${ }^{8}$ R. K. Osborn and S. Yip, The Foundations of Neutron Transport Theory (Gordon and Breach Science Publishers, New York, to be published). liquid, or a gas. However, the present calculation will be explicitly restricted to large systems in solid phase.

\section{THEORY}

The total energy $(\mathfrak{H C})$ of the system is divided into two parts,

$$
\mathfrak{F}=H_{0}+V ;
$$

each of which is further subdivided as

and

$$
\begin{gathered}
H_{0}=H^{\gamma}+H^{N}+H^{C}+V_{0}^{N C}, \\
V=V^{\gamma}+V_{\tau}^{N C} .
\end{gathered}
$$

The energy, $H^{\gamma}$, is that of the free radiation field; $H^{N}$ is that part of the energy of the decaying nucleus which is independent of its environment, $H^{C}$ is the energy of the crystal, including the kinetic and potential energy of the decaying nucleus which depends upon the position and velocity of its center of mass; and $V_{0}{ }^{N C}$ is the energy of interaction of the decaying nucleus with any stationary electric and magnetic fields that may be present. The energy $V^{\gamma}$ arises from the interaction of the decaying nucleus with the ambient radiation field, whereas $V_{\tau}{ }^{N C}$ is the energy of interaction between the decaying nucleus and the environmental atoms which depends upon the displacement of the latter from their equilibrium positions. Thus $V^{\gamma}$ is the interaction responsible for the emission of the observed gamma rays as well as a radiative widening and shifting of the nuclear levels involved in the cascade, whereas $V_{\tau}{ }^{N C}$ produces a further widening and shifting of the nuclear energy levels-due to dynamic coupling with the environment-especially those populated after the emission of the first gamma ray. It is this latter shifting and broadening which introduces an explicit temperature dependence into the calculations of the perturbation factor in the angular correlation.

The states of the system will be presumed char- 
acterized by the eigenfunctions of $H_{0}$. These eigenfunctions are constructed according to

$$
\begin{gathered}
H^{\gamma}|\eta\rangle=E_{\eta}|\eta\rangle, \\
\left(H^{N}+V_{0}^{N C}\right)|q I K\rangle=E_{q I K}|q I K\rangle,
\end{gathered}
$$

and

$$
H^{C}|\alpha\rangle=E_{\alpha}|\alpha\rangle .
$$

The eigenfunctions $|\eta\rangle$ are the eigenfunctions of the photon number operator; thus $E_{\eta}=\sum_{\pi \lambda} \hbar c \pi \eta(\pi \lambda)$, where $\pi$ is a photon wave number and $\eta(\pi, \lambda)$ is the number of photons with wave number $\pi$ and polarization $\lambda$ in the radiation state specified by $|\eta\rangle$. The functions $|q I K\rangle$ are linear combinations of the angular-momentum eigenfunctions, $|q I m\rangle$, of the decaying nucleus. The label $(q)$ represents a set of labels sufficient to specify the internal states of the radioactive nucleus with the exception of its total angular momentum and $z$ component. The eigenfunctions of $H_{0}$, corresponding to the eigenvalue $E_{\beta}$, are then taken to be

$$
|\beta\rangle=|q I K\rangle|\alpha\rangle|\eta\rangle \text {. }
$$

With this representation the correlation function descriptive of a direction-direction correlation measurement can be expressed as

$$
W\left(\boldsymbol{\Omega}_{2}, \boldsymbol{\Omega}_{1}\right)=\sum_{\lambda_{1} \lambda_{2}} \sum_{\beta_{i} \beta_{f}} P\left(\beta_{i}\right) \frac{\left|\left\langle\beta_{i}|U(\tau)| \beta_{f}\right\rangle\right|^{2}}{\tau} .
$$

The eigenfunctions $\left|\beta_{i}\right\rangle,|\beta\rangle$, and $\left|\beta_{f}\right\rangle$ belong to the orthonormal complete set of eigenfunctions $\{|\beta\rangle\}$ which satisfy $H_{0}|\beta\rangle=E_{\beta}|\beta\rangle$ and correspond to the initial state, the intermediate state and the final state of the radioactive nucleus in the cascade. The quantity $U(\tau)$ is the time-evolution operator and is given by $U(\tau)$ $=\exp (-i \mathcal{H C} \tau / \hbar)$ where $\tau$ is a small time interval (but large compared to relevant lifetimes). $P\left(\beta_{i}\right)$ is the probability that the system is in an eigenstate $\left|\beta_{i}\right\rangle$. By means of the damping theory the off-diagonal matrix elements of $U(\tau)$ can be calculated as, ${ }^{8}$

$$
\begin{array}{r}
\frac{\left|\left\langle\beta_{i}|U(\tau)| \beta_{f}\right\rangle\right|^{2}}{\tau}=\left|\sum_{\beta \neq \beta_{i}, \beta_{f}} \frac{V^{\gamma_{\beta_{i} \beta}} V^{\gamma_{\beta \beta_{f}}}}{E_{\beta}-E_{\beta_{i}}+\frac{1}{2} \hbar \gamma_{\beta}\left(-i E_{\beta_{i}}\right)}\right|^{2} \\
\times \delta\left(E_{\beta_{i}}-E_{\beta_{f}}\right),
\end{array}
$$

where

$$
\frac{1}{2} \hbar \gamma_{\beta}\left(-i E_{\beta_{i}}\right)=V_{\beta \beta}+\lim _{\lambda \rightarrow 0} \sum_{\beta_{m} \neq \beta} \frac{\left|V_{\beta_{m} \beta}\right|^{2}}{E_{\beta_{i}}-E_{\beta_{m}}+i \lambda} .
$$

In deriving (2.6) the width and shift of the initial and final states of the decaying nucleus have explicitly been ignored.

Combining Eqs. (2.5) and (2.6) one has

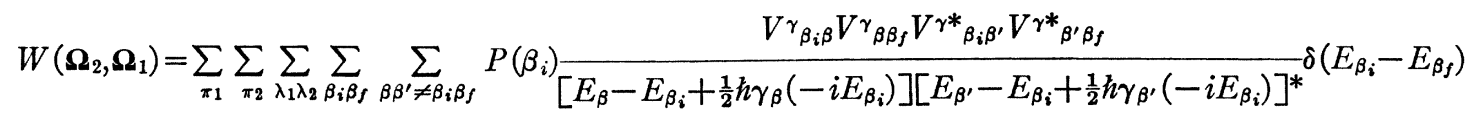

where $\sum_{\pi}$ denotes the summation of all possible energies of the $\gamma$ ray. It will be shown in the next section that Eq. (2.7) can be displayed as:

$$
\frac{1}{2} h \gamma_{\beta}\left(-i E_{\beta_{i}}\right)=S_{\beta}\left(V_{\tau}^{N C}\right)-\frac{1}{2} i \hbar\left\{\Gamma_{\beta}\left(V_{\tau}^{N C}\right)+\tau_{N}^{-1}\right\},
$$

where $S_{\beta}\left(V_{\tau}{ }^{N C}\right)$ and $\frac{1}{2} \hbar \Gamma_{\beta}\left(V_{\tau}^{N C}\right)$ can be interpreted as the nuclear-energy-level shift and width corresponding to an intermediate state $|\beta\rangle$ due to the dynamic part of the coupling, and $\tau_{N}$ is the experimentally determined radiative mean life of the radioactive nucleus in the intermediate state.

It has been shown that ${ }^{8} V^{\gamma}$ can be separated into two parts when the nuclear momentum is small com- pared to the nucleonic momentum, i.e.,

$$
V^{\gamma}=e^{-i \pi \cdot \mathbf{R}} V(\gamma)
$$

where $V(\gamma)$ depends only on the internal degrees of freedom of the radioactive nucleus and the photon field. The vector $\mathbf{R}$ is the position vector of the center of mass of the radioactive nucleus and $\pi$ is the wave vector of the emitted photon. By making the expansion

$$
|q I K\rangle \equiv|\kappa\rangle=\sum_{m}|\operatorname{Im}\rangle\langle\operatorname{Im} \mid \kappa\rangle
$$

and using Racah's technique the perturbed correlation function of Eq. (2.8), for the case in which the nuclearenergy-level width is much less than the level splitting, can be calculated as;

$$
W\left(\boldsymbol{\Omega}_{2}, \boldsymbol{\Omega}_{1}\right)=\sum_{k_{1} k_{2}} \sum_{N_{1} N_{2}} A_{k_{1}}(1) A_{k_{2}}(2)\left[\left(2 k_{1}+1\right)\left(2 k_{2}+1\right)\right]^{-1 / 2} \bar{G}_{k_{1} k_{2}}{ }^{N_{1} N_{2}} Y_{k_{1}}{ }^{N_{1} *}\left(\theta_{1} \varphi_{1}\right) Y_{k_{2}}{ }^{N_{2}}\left(\theta_{2} \varphi_{2}\right),
$$

where

$$
\bar{G}_{k_{1} k_{2}} N_{1} N_{2} \equiv \sum_{\beta_{i}} P\left(\beta_{i}\right) G_{k_{1} k_{2}} N_{1 N_{2}}
$$


and

$$
\begin{aligned}
& \left.G_{k_{1} k_{2}} N_{1} N_{2}=\sum_{m m^{\prime}} \sum_{\kappa \kappa^{\prime}}(-1)^{2 I+m^{\prime}+m}\left[\left(2 k_{1}+1\right)\left(2 k_{2}+1\right)\right)\right]^{1 / 2}\left(\begin{array}{ccc}
I & I & k_{1} \\
m^{\prime \prime \prime} & -m^{\prime} & N_{1}
\end{array}\right)\left(\begin{array}{ccc}
I & I & k_{2} \\
m^{\prime \prime} & -m & N_{2}
\end{array}\right)\langle\operatorname{Im} \mid \kappa\rangle\left\langle\kappa \mid I m^{\prime}\right\rangle \\
& \times\left\langle\operatorname{Im}^{\prime \prime \prime} \mid \kappa^{\prime}\right\rangle\left\langle\kappa^{\prime} \mid I m^{\prime \prime}\right\rangle\left\{\left[1+\frac{1}{2} \tau_{N}\left(\Gamma_{\kappa}\left(V_{\tau}^{N C}\right)+\Gamma_{\kappa^{\prime}}\left(V_{\tau}^{N C}\right)\right]-\frac{i\left(E_{\kappa}-E_{\kappa^{\prime}}\right) \tau_{N}}{\hbar}\right.\right. \\
& \left.\times\left[1+\frac{S_{\kappa}\left(V_{\tau}^{N C}\right)-S_{\kappa^{\prime}}\left(V_{\tau}^{N C}\right)}{E_{\kappa}-E_{\kappa^{\prime}}}\right]\right\}^{-1}
\end{aligned}
$$

and

$$
E_{\kappa}-E_{\kappa^{\prime}}=\sum_{m m^{\prime}}\left[\left\langle\operatorname{Im}^{\prime} \mid \kappa\right\rangle\langle\kappa \mid \operatorname{Im}\rangle-\left\langle\operatorname{Im}^{\prime} \mid \kappa^{\prime}\right\rangle\left\langle\kappa^{\prime} \mid \operatorname{Im}\right\rangle\right]\left\langle\operatorname{Im}\left|V_{0}{ }^{N C}\right| \operatorname{Im} m^{\prime}\right\rangle .
$$

The perturbed directional correlation function of Eq. (2.12) has exactly the same form of Steffen and Frauenfelder's Eq. (8), ${ }^{9}$ except the perturbation factor in the present calculation is given by Eq. (2.13) which is a timeintegrated and thermally averaged perturbation factor. The function $\bar{G}_{k_{1} k_{2}}{ }^{N_{1} N_{2}}$ is the averaged perturbation factor over the initial state of the system.

If the system is initially in thermal equilibrium, and in doing the thermal average we follow an approximation which is made by replacing the average of a ratio of two functions by the ratio of the average function, ${ }^{10}$ then Eq. (2.13) can be written

$$
\begin{aligned}
\bar{G}_{k_{1} k_{2}} N_{1} N_{2}=\sum_{m m^{\prime}} \sum_{\kappa k^{\prime}}(-1)^{2 I+m^{\prime}+m}\left[\left(2 k_{1}+1\right)\left(2 k_{2}+1\right)\right]^{1 / 2}\left(\begin{array}{ccc}
I & I & k_{1} \\
m^{\prime \prime \prime} & -m^{\prime} & N_{1}
\end{array}\right)\left(\begin{array}{ccc}
I & I & k_{2} \\
m^{\prime \prime} & -m & N_{2}
\end{array}\right)\langle I m \mid \kappa\rangle\left\langle\kappa \mid I m^{\prime}\right\rangle \\
\times\left\langle\operatorname{Im}^{\prime \prime \prime} \mid \kappa^{\prime}\right\rangle\left\langle\kappa^{\prime} \mid I m^{\prime \prime}\right\rangle\left\{\left[1+\frac{1}{2} \tau_{N}\left(\left\langle\Gamma_{\kappa}\left(V_{\tau}^{N C}\right)\right\rangle_{T}+\left\langle\Gamma_{\kappa^{\prime}}\left(V_{\tau}^{N C}\right)\right\rangle_{T}\right)\right]-\frac{i\left(E_{\kappa}-E_{\kappa^{\prime}}\right) \tau_{N}}{\hbar}\right. \\
\\
\left.\times\left[1+\frac{\left\langle S_{\kappa}\left(V_{\tau} \tau^{N C}\right)\right\rangle_{T}-\left\langle S_{\kappa^{\prime}}\left(V_{\tau}{ }^{N C}\right)\right\rangle_{T}}{E_{\kappa}-E_{\kappa^{\prime}}}\right]\right\}^{-1} .
\end{aligned}
$$

The quantities $\left\langle S_{\kappa}\left(V_{\tau}{ }^{N C}\right)\right\rangle_{T}$ and $\frac{1}{2} h\left\langle\Gamma_{\kappa}\left(V_{\tau}{ }^{N C}\right)\right\rangle_{T}$ are, respectively, the averaged nuclear-energy-level shift and width due to $V_{\tau}{ }^{N C}$ over the initial state of the system and will be explicitly calculated in the next section. The factors $A_{k_{1}}(1)$ and $A_{k_{2}}(2)$ depend on the multipolarities of the consecutive gamma rays and the spin of the successive nuclear levels and are independent of the extranuclear perturbing fields. A detailed discussion and calculations of these two factors have been given by Biedenharn and Rose. ${ }^{11}$ The influence of the extranuclear fields on the angular correlation is entirely contained in the averaged perturbation factor $\bar{G}_{k_{1} k_{2}}{ }^{N_{1} N_{2}}$. The probability function $P\left(\beta_{i}\right)$ in Eq. (2.13) is a diagonal element of the density matrix which contains all the information of the whole physical system at the initial state.

Particularly, the average over the crystal states will take into account all the effects on the correlation due to the lattice vibrations and all kinds of lattice imper-

\footnotetext{
${ }^{9}$ R. M. Steffen and H. Frauenfelder, in Perturbed Angular Correlations, edited by E. Karlsson, E. Matthias, and K. Siegbahn (North-Holland Publishing Company, Amsterdam, 1964), Chap. I. ${ }^{10}$ A. Ziya Akcasu, University of Michigan Technical Report (unpublished).

${ }^{11}$ L. C. Biedenharn and M. E. Rose, Rev. Mod. Phys. 25, 729 (1953).
}

fections due to, for instance, the nuclear recoils, the radioactive decays and the variations of the crystalline field within the crystal and so on. Recently Matthias, Schneider, and Steffen ${ }^{12}$ have pointed out that a normal distribution of the electric interaction frequency can be assumed in order to estimate the effects on the perturbation factor due to the variations of the axially symmetric electric field gradients at the different sites of the nuclei in an imperfect crystal lattice. The frequency distribution effects will also be contained in the probability function in the present theory. However, our knowledge about all the imperfections and the distributions of these imperfections in a crystal lattice is quite limited and it becomes very difficult for one to investigate all these effects at the same time. In the present calculation, the effects of the lattice vibrations on the perturbation factor are investigated and the result shows a temperature dependence of the perturbation factor.

If the level shift is negligible and the nuclear relaxation times are long compared with the life time of the nucleus in the intermediate state, Eq. (2.12) reduces to

${ }^{12}$ E. Matthias, W. Schneider, and R. M. Steffen, Phys. Letters 4, 41 (1963). 
Alder's ${ }^{13}$ and Abragam and Pound's ${ }^{14}$ results for the case of static fields.

For vanishing perturbation, Eq. (2.16) reduces to

$$
\bar{G}_{k_{1} k_{2}} N_{1} N_{2}=\delta_{k_{1} k_{2}} \delta_{N_{1} N_{2}} .
$$

Substituting Eq. (2.17) into Eq. (2.12), one can show that Eq. (2.12) reduces to the well-known unperturbed correlation function which can be expressed as the sum of even Legendre polynomials.

When the source is in an external field of axial symmetry, e.g., an external magnetic field, one can choose the symmetry axis as the quantization axis and the perturbed correlation functions of Eqs. (2.12) and (2.13) can be reduced to ${ }^{15}$;

$W\left(\mathbf{\Omega}_{\mathbf{2}}, \mathbf{\Omega}_{\mathbf{1}}\right)$

$$
\begin{aligned}
& =\sum_{k_{1} k_{2}} \sum_{N} A_{k_{1}}(1) A_{k_{2}}(2)\left[\left(2 k_{1}+1\right)\left(2 k_{2}+1\right)\right]^{-1 / 2} \\
& \times{G_{k_{1} k_{2}}}^{N N} Y_{k_{1}}{ }^{N *}\left(\theta_{1}, \varphi_{1}\right) Y_{k_{2}}{ }^{N}\left(\theta_{2}, \varphi_{2}\right),
\end{aligned}
$$

where

$$
\begin{aligned}
\bar{G}_{k_{1} k_{2}}{ }^{N N}= & \sum_{m}\left[\left(2 k_{1}+1\right)\left(2 k_{2}+1\right)\right]^{1 / 2} \\
& \times\left(\begin{array}{ccc}
I & I & k_{1} \\
m^{\prime} & -m & N
\end{array}\right)\left(\begin{array}{ccc}
I & I & k_{2} \\
m^{\prime} & -m & N
\end{array}\right) \\
& \times\left\{1-i\left(E_{m^{\prime}}-E_{m}\right) \tau_{N} / \hbar\right\}^{-1} .
\end{aligned}
$$

If the first detector is placed along the symmetric field, Eqs. (2.18) and (2.19) can be reduced to the unperturbed angular correlation function. This is a well-known result, that the correlation is unperturbed by such a coupling if either one of the two radiations is emitted along the axially symmetric field. ${ }^{14}$

\section{THE PERTURBATION FACTOR- SINGLE CRYSTAL}

Extranuclear perturbations experienced by a radioactive nucleus sitting in a lattice site may be the atomic hyperfine interaction, the nuclear magnetic dipole-dipole interactions with the surrounding nuclei, or the magnetic interaction with an externally applied magnetic field, and the nuclear electric quadrupole interaction with the crystalline field. In our discussions we will focus our attention on the electric quadrupole interaction. By substituting $V=V^{\gamma}+V_{\tau}{ }^{\mathrm{EQ}}$ into Eq. (2.7) $\left(V_{\tau}{ }^{\mathrm{EQ}}\right.$ denotes the dynamic part of the electric quadrupole interactions), the thermally averaged shift and width function can be expressed as

$$
\left\langle\frac{1}{2} \hbar \gamma_{\kappa}\left(-i E_{\beta_{i}}\right)\right\rangle_{T}=\left\langle\frac{1}{2} \hbar \gamma_{\kappa}\left(V_{\tau}\right)^{\mathrm{EQ}}\right\rangle_{T}+\frac{1}{2} \hbar \gamma_{\kappa}\left(V^{\gamma}\right)
$$

${ }^{13}$ K. Alder, Helv. Phys. Acta 25, 235 (1952).

${ }^{14}$ A. Abragam and R. V. Pound, Phys. Rev. 92, 943 (1953).

${ }^{15} \mathrm{H}$. Frauenfelder and R. M. Steffen, in Alpha-, Beta- and Gamma Ray Spectroscopy, edited by K. Siegbahn (North-Holland Publishing Company, Amsterdam, 1965), Chap. XIX A. where

$$
\begin{aligned}
& \left\langle\frac{1}{2} h \gamma_{\kappa}\left(V_{\tau}{ }^{\mathrm{EQ}}\right)\right\rangle_{T} \\
& =\lim _{\lambda \rightarrow 0} \sum_{\beta_{i}} P\left(\beta_{i}\right) \sum_{\beta^{\prime} \neq \beta} \frac{\left|\left\langle\beta^{\prime}\left|V_{\tau}^{\mathbf{E Q}}\right| \beta\right\rangle\right|^{2}}{E_{\beta_{i}}-E_{\beta^{\prime}}+i \lambda},
\end{aligned}
$$

and

$$
\frac{1}{2} h \gamma_{\kappa}\left(V^{\gamma}\right)=\lim _{\lambda \rightarrow 0} \sum_{\beta_{m} \neq \beta} \frac{\left|\left\langle\beta_{m}\left|V^{\gamma}\right| \beta\right\rangle\right|^{2}}{E_{\beta_{i}}-E_{\beta_{m}}+i \lambda} .
$$

In Eq. (3.1) we have neglected the changes of states of the lattice system due to the emission of the cascade photons. The quantity $\frac{1}{2} \hbar \gamma_{\kappa}\left(V^{\gamma}\right)$ can be expressed ${ }^{8}$ as

$$
\frac{1}{2} \hbar \gamma_{\kappa}\left(V^{\gamma}\right)=S_{\kappa}\left(V^{\gamma}\right)-\frac{1}{2} i \hbar \Gamma_{\kappa}\left(V^{\gamma}\right),
$$

where $S_{\kappa}\left(V^{\gamma}\right)$ is the radiative shift function and for our purpose we will only note its existence and will not be concerned with its effects. The quantity $\frac{1}{2} h \Gamma_{\kappa}\left(V^{\gamma}\right)$ is the radiative width function and is independent of the substates of the intermediate and the final nuclear states but depends on the angular momenta $I$ and $I_{f}$ and also on the type of transitions. In our calculation we will let $\Gamma_{\kappa}\left(V^{\gamma}\right)=\tau_{N}{ }^{-1}$; here $\tau_{N}$ is the experimentally determined life time of the radioactive nucleus in the intermediate state. Then Eq. (3.4) reduces to

$$
\frac{1}{2} \hbar \gamma_{\kappa}\left(V^{\gamma}\right)=-i \hbar / 2 \tau_{N}
$$

The electric quadrupole interaction Hamiltonian can be expressed as

$$
V^{\mathrm{EQ}}=\sum_{\zeta=-2}^{2}(-1)^{\zeta} Q_{\zeta}^{(2)} V_{-\zeta}^{(2)}
$$

where $Q_{\zeta}^{(2)}$ and $V_{-\zeta}^{(2)}$ are, respectively, the nuclear electric quadrupole moment tensor and the field tensor and can be written as

$$
\begin{aligned}
& Q_{\zeta}^{(2)}=\sum_{p} e_{p} r_{p}^{2} Y_{2}^{\zeta}\left(\theta_{p}, \varphi_{p}\right), \\
& V_{-\zeta}^{(2)}=\sum_{i} \frac{\rho_{i}}{r_{c i}{ }^{3}} Y_{2}^{-\zeta}\left(\theta_{c i}, \varphi_{c i}\right) .
\end{aligned}
$$

The charge $e_{p}$ is the nucleonic charge in the nucleus at the position $\mathbf{r}_{p}$ with respect to the center of the radioactive nucleus. In Eq. (3.8) we have assumed that the electrostatic field is caused by the point charges in the lattice system and $\rho_{i}$ is the effective point charge at the $i$ th lattice point located at $\mathbf{r}_{c i}$ which is the relative position vector between the radioactive nucleus and lattice point. The vibration of a nucleus in a crystal can be described by a displacement vector $\mathbf{u}$ from its equilibrium position and the relative vector becomes $\mathbf{r}_{c i}$ $=\mathbf{a}_{0 c i}-\mathbf{u}_{c i}$; here $\mathbf{a}_{0 c i}$ is the relative equilibrium position vector between the radioactive nucleus and the $i$ th lattice point, and $\mathbf{u}_{c i}$ is the relative displacement vector between these two nuclei. When the temperature is far below the melting point of the crystal one has $u_{c i} \ll<a_{0 c i}$ and then the field tensors can be expanded at the equi- 
librium positions as ${ }^{16}$;

$$
\begin{aligned}
V_{-\zeta}^{(2)}=V_{-\zeta}^{(2)}(0)+\frac{15}{2} \sum_{i} & \sum_{\mu \nu} \frac{\rho_{i}}{a^{5}{ }_{0 c i}} Y_{2}^{-\zeta}\left(\theta_{0 c i}, \varphi_{c c i}\right) \\
& \times \eta_{i \mu} \eta_{i \nu} u_{\mu}(i) u_{\nu}(i)+\cdots,
\end{aligned}
$$

where $V_{-\zeta^{(2)}}(0)$ is an irreducible component of the field tensor evaluated at the equilibrium positions, $\eta_{i \mu}$ and $\eta_{i v}$ are, respectively, the directional cosines of $\mathbf{a}_{0 c i}$ in the $\mu$ th and $\nu$ th directions and $u_{\mu}(i)$ is the $\mu$ th component of the relative displacement $\mathbf{u}_{c i}$. By using the normal mode expansion of the displacement, the vector $\mathbf{u}(i)$ $=\mathbf{u}_{c}\left(\mathbf{r}_{0 c}\right)-\mathbf{u}_{i}\left(\mathbf{r}_{0 i}\right)$ can be expressed as a function of the phonon creation and annihilation operators ${ }^{17}$;

$$
\begin{aligned}
& \mathbf{u}(i)=\sum_{\mathbf{q} j}\left[\frac{\hbar}{2 M_{c} \omega(\mathbf{q} j)}\right]^{1 / 2} \mathbf{e}(\mathbf{q} j)\left\{a^{\dagger}(\mathbf{q} j) e^{-i \mathbf{q} \cdot \mathbf{r}_{0 c}}\right. \\
& \times\left(1-\epsilon_{c i} e^{-i \mathbf{q} \cdot \mathbf{a} 0 c i}\right)+a(\mathbf{q} j) e^{i \mathbf{q} \cdot \mathbf{r}_{0 c}} \\
&\left.\times\left(1-\boldsymbol{\epsilon}_{c i} e^{i \mathbf{q} \cdot \mathbf{a}_{0 c} i}\right)\right\}
\end{aligned}
$$

where $\epsilon_{c i}=M_{c} / M_{i}$ is the ratio of the mass of the radioactive nucleus to the $i$ th nucleus in the crystal. In the case of a single crystal, $\epsilon_{c i}$ is the isotopic factor. When the wavelength of a phonon is appreciably larger than the interatomic spacing, we make the approximation $\exp \left(-i \mathbf{q} \cdot \mathbf{a}_{0 c i}\right) \simeq 1-i q a_{0 c i}\left(\hat{q} \cdot \hat{a}_{0 c i}\right)$. This approximation will considerably simplify our calculations. Although it may break down at the upper end of the Debye spectrum, it will not affect the results appreciably. ${ }^{18}$ Using Eqs. (3.6), (3.9), and (3.10), the electric quadrupole coupling Hamiltonian can be expressed as;

$$
\begin{aligned}
& V^{\mathrm{EQ}}=V_{0}^{\mathrm{EQ}}+V_{\tau}^{\mathrm{EQ}}, \\
& \left\langle S_{\kappa}\left(V_{\tau}{ }^{\mathrm{EQ}}\right)\right\rangle_{T}=\left(\frac{15 \hbar V}{4 M_{c} v^{3}}\right)^{2}\left|\left\langle I\left\|Q^{(2)}\right\| I\right\rangle\right|^{2} \sum_{\zeta=-2}^{2} \sum_{\alpha^{\prime} \neq \kappa} \sum_{m, m^{\prime}} \sum_{i}^{(n, n)} \sum_{\mu \nu}\left(\frac{\rho_{i}}{a_{0 c i}{ }^{3}}\right)^{2}\left(\begin{array}{ccc}
I & 2 & I \\
-m^{\prime} & \zeta & m
\end{array}\right)^{2} Y_{2}{ }^{-\zeta}\left(\theta_{0 c i}, \varphi_{0 c i}\right) \\
& \times\left|\langle I m \mid \kappa\rangle\left\langle\kappa^{\prime} \mid I m^{\prime}\right\rangle\right|{ }^{2} \eta_{i \mu^{2}}{ }^{2} \eta_{i \nu}{ }^{2} P \int_{\omega_{\kappa \kappa^{\prime}}}^{\omega M} d \omega \int_{0}^{\omega M-\omega_{\kappa \kappa^{\prime}}} d \omega^{\prime} \int_{\Omega(\hat{q})} d \Omega \int_{\Omega^{\prime}\left(\hat{q}^{\prime}\right)} d \Omega^{\prime} \omega \omega^{\prime}\left[d_{c i}{ }^{2}+\epsilon_{c i^{2}}{ }^{2}{ }_{\tau^{2}}{ }^{2}\left(\hat{q} \cdot \hat{a}_{0 c i}\right)^{2}\right] \\
& \times\left[d_{c i}{ }^{2}+\epsilon_{c i}{ }^{\omega^{\prime 2}} \frac{{ }^{2}}{v^{2}}\left(\hat{q}^{\prime} \cdot \hat{a}_{0 c i}\right)^{2}\right] \frac{n_{T}(\omega)\left[n_{T}\left(\omega^{\prime}\right)+1\right]}{\hbar \omega-\hbar \omega^{\prime}-\hbar \omega_{\kappa k^{\prime}}}, \\
& \left\langle\Gamma_{\kappa}\left(V_{\tau} \mathrm{EQ}\right)\right\rangle_{T}=2 \pi h\left(15 V / 4 M_{c} v^{3}\right)^{2}\left|\left\langle I\left\|Q^{(2)}\right\| I\right\rangle\right|^{2} \sum_{\zeta=-2}^{2} \sum_{\kappa^{\prime} \neq \kappa} \sum_{m m^{\prime}} \sum_{i}^{(n, n)} \sum_{\mu \nu}\left(\frac{\rho_{i}}{a_{0 c i}{ }^{3}}\right)^{2}\left(\begin{array}{ccc}
I & 2 & I \\
-m^{\prime} & \zeta & m
\end{array}\right)^{2} Y_{2}^{-\zeta}\left(\theta_{0 c i}, \varphi_{0 c i}\right) \\
& \times\left|\langle\operatorname{Im} \mid \kappa\rangle\left\langle\kappa^{\prime} \mid \operatorname{Im}^{\prime}\right\rangle\right|{ }^{2} \eta_{i \mu^{2}}{ }^{2} \eta_{\nu \nu^{2}} \int_{\omega_{\kappa \kappa^{\prime}}}^{\omega} d \omega \int_{0}^{\omega_{M}-\omega_{\kappa \kappa^{\prime}}} d \omega^{\prime} \int_{\Omega(\hat{q})} d \Omega \int_{\Omega^{\prime}\left(\hat{q}^{\prime}\right)} d \Omega^{\prime} \omega \omega^{\prime}\left[d_{c i}{ }^{2}+\epsilon_{c i}{ }^{2}{ }_{v^{2}}{ }^{2}\left(\hat{q} \cdot \hat{a}_{0 c i}\right)^{2}\right] \\
& \times\left[d_{c i}{ }^{2}+\epsilon_{c i}{ }^{{ }^{2}}{ }^{\prime 2}\left(\hat{q}^{\prime} \cdot \hat{a}_{0 c i}\right)^{2}\right] n_{T}(\omega)\left[n_{T}\left(\omega^{\prime}\right)+1\right] \delta\left(\hbar \omega-\hbar \omega^{\prime}-\hbar \omega_{\kappa \kappa^{\prime}}\right),
\end{aligned}
$$

with

and

$$
\begin{aligned}
V_{\tau}{ }^{\mathrm{EQ}}= & \left(\frac{15 \hbar}{4 M_{c}}\right) \sum_{\zeta} \sum_{i}^{(n, n)} \sum_{\mu \nu} \sum_{\mathbf{q} \mathbf{q}^{\prime}}(-1)^{\zeta}\left(\frac{\rho_{i}}{a_{0 c i}{ }^{3}}\right) \eta_{i \mu} \eta_{i \nu} \\
& \times\left[\omega(\mathbf{q} \mu) \omega\left(\mathbf{q}^{\prime} \nu\right)\right]^{-1 / 2}\left[d_{c i}-i \epsilon_{c i} q\left(\hat{q} \cdot \hat{a}_{0 c i}\right)\right] \\
& \times\left[d_{c i}+i \epsilon_{c i} q^{\prime}\left(\hat{q}^{\prime} \cdot \hat{a}_{0 c i}\right)\right] Y_{2}^{-\zeta}\left(\theta_{0 c i}, \varphi_{0 c i}\right) \\
& \times Q_{\zeta}^{(2)} a^{\dagger}\left(\mathbf{q}^{\prime} \nu\right) a(\mathbf{q} \mu) e^{i\left(\mathbf{q}-\mathbf{q}^{\prime}\right) \cdot \mathrm{r}_{00}}
\end{aligned}
$$

where $V_{0}{ }^{\mathrm{EQ}}$ represents the static electric quadrupole coupling Hamiltonian when the crystal is considered as a rigid lattice system and $V_{\tau}{ }^{\mathrm{EQ}}$ describes the dynamic part of the electric quadrupole coupling induced by the Raman processes. For the summation of $\mathbf{q}$ in Eq. (3.13), one should sum over all the possible values of $\mathbf{q}$ in the first Brillouin zone of the reciprocal lattice space. However, for our purpose it is sufficient to take Debye's assumption that the vibrational frequency spectrum is a continuum and the sound propagation velocity $\mathbf{v}$ is independent of the direction of propagation and of the polarization of the wave. Furthermore the total number of nuclei in a crystal is much greater than unity, so one can replace the first Brillouin zone by a sphere. Then by substituting Eq. (3.13) into Eq. (3.2) and using the relation

$$
\lim _{\lambda \rightarrow 0}(i \lambda+y)^{-1}=P(y)^{-1}-i \pi \delta(y),
$$

where $P(y)^{-1}$ is the principal value of $y^{-1}$, the quantity $\left\langle\frac{1}{2} \hbar \gamma_{\kappa}\left(V_{\tau}{ }^{\mathrm{EQ}}\right)\right\rangle_{T}$ can be expressed as

$$
\left\langle\frac{1}{2} \hbar \gamma_{\kappa}\left(V_{\tau}^{\mathrm{EQ}}\right)\right\rangle_{T}=\left\langle S_{\kappa}\left(V_{\tau}^{\mathrm{EQ}}\right)\right\rangle_{T}-i\left\langle\frac{1}{2} \hbar \Gamma_{\kappa}\left(V_{\tau}^{\mathrm{EQ}}\right)\right\rangle_{T},
$$

${ }^{16} \mathrm{M}$. Born and K. Huang, Dynamical Theory of Crystal Lattices (Oxford University Press, New York, 1954). ${ }^{17}$ A. Maradudin, E. W. Montroll, and G. H. Weiss, Solid State Physics, edited by F. Seitz and D. Turnbull (Academic Press Inc.,
New York, 1963), Suppl. 3.

${ }^{18}$ A. Abragam, The Principles of Nuclear Magnetism (Oxford University Press, New York, 1961). 
where $\hbar \omega_{\mathrm{xk}^{\prime}}$ denotes $E_{\mathrm{k}}-E_{\mathrm{k}^{\prime}}$, and is the nuclear-energylevel splittings due to the static part of couplings and can be expressed as

$$
\begin{aligned}
E_{\kappa}-E_{\kappa^{\prime}}= & \sum_{\zeta=-2}^{2} \sum_{m m^{\prime}}\left\{\left\langle I m^{\prime} \mid \kappa\right\rangle\langle\kappa \mid I m\rangle-\left\langle I m^{\prime} \mid \kappa^{\prime}\right\rangle\left\langle\kappa^{\prime} \mid I m\right\rangle\right\} \\
& \times(-1)^{I-m}\left(\begin{array}{ccc}
I & 2 & I \\
-m & \zeta & m^{\prime}
\end{array}\right) V_{-\zeta^{(2)}}(0) \\
& \times\left\langle I\left\|Q^{(2)}\right\| I\right\rangle .
\end{aligned}
$$

The quantity $n_{T}(\alpha)=\sum_{n(\alpha)} P(n(\alpha)) n(\alpha)$ is the thermally averaged phonon occupation number in the crystal, and $d_{c i}$ stands for $\left(1-\epsilon_{c i}\right) / a_{0 c i}$ and $V$ is the volume of the crystal. For a Raman process the frequencies of the two phonons $\omega$ and $\omega^{\prime}$ satisfy $\hbar \omega-\hbar \omega^{\prime}=\hbar \omega_{\kappa k^{\prime}}$ and $\omega$ can take all the values inside the frequency spectrum from $\omega_{k x^{\prime}}$ to $\omega_{M}$, where $\omega_{M}$ is the maximum frequency of the Debye spectrum.

The electric-field tensor appearing in Eqs. (3.15) and (3.16) is produced by the $i$ th lattice point charge in the neighborhood of the radioactive nucleus in the absence of the nucleus and is referred to as the direct field. This point-charge model cannot take into account the shielding, antishielding, and covalent effects. In order to compensate this point one introduces an adjustable multiplication parameter $\xi_{i}$ such that the effective point charge of a lattice point is taken to be $\rho_{i}=\xi_{i} e$. This parameter $\xi_{i}$ is determined by comparing the experimental results and theoretical calculations in the microwave techniques..$^{19,20}$ Combining Eqs. (3.15), (3.16), and (2.16) one has the perturbation factor which describes the lattice vibration effects on the angular correlation. It is due to this effect that a temperature dependence of the perturbed angular correlation can be established, and this will be shown in Sec. V.

\section{THE ROTATIONAL DEPENDENCE}

It has been shown experimentally by the Zürich group $^{2-4}$ that the anisotropy of the $\gamma-\gamma$ angular correlation is a function of the orientations of the symmetric axis of the crystalline field with respect to the detectors. Recently Paul and Brummer, ${ }^{21}$ Alder et al. ${ }^{22}$ have investigated the rotational dependence of the anisotropy functions for the case of axially symmetric fields. In the following we will investigate the effects on the rotational pattern of the angular correlation by the

asymmetric crystalline field by taking into account the lattice vibrations.

We let $x y z$ be an arbitrarily chosen laboratory coordinate system, $X Y Z$ be a fixed coordinate system in the crystal and $X^{*} Y^{*} Z^{*}$ be the principal axis coordinates system of the crystalline field. The Euler angles between the $X Y Z$ and $X^{*} Y^{*} Z^{*}$ coordinate systems are taken to be $(\alpha \beta \gamma)$ which can be determined by EPR and NMR techniques. The Euler angles between the crystal coordinates $X Y Z$ and the laboratory coordinates $x y z$ are taken to be $(\Phi \Theta \Omega)$. The Euler angles are defined in a right-handed coordinate system and the rotational relations between these three sets of coordinates can be expressed as;

$$
\Sigma^{*}\left(X^{*} Y^{*} Z^{*}\right) \stackrel{R(a \beta \gamma)}{\longrightarrow} \Sigma^{(\mathrm{II})}(X Y Z) \stackrel{R(\Phi \Theta \Omega)}{\longrightarrow} \Sigma^{(\mathrm{I})}(x y z) .
$$

Then an irreducible field tensor of rank $2,{ }^{*} V^{(2)}$ in the principal-axis coordinate system $\Sigma^{*}$ can be transformed into the laboratory coordinate system by

$$
{ }^{(1)} V_{-\zeta}^{(2)}=\sum_{\rho, r} D_{r \rho}^{(2)}(\alpha \beta \gamma) D_{\rho-\zeta}^{(2)}(\Phi \Theta \Omega)^{*} V_{r}^{(2)},
$$

where ${ }^{(\mathrm{I})} V_{-\zeta^{(2)}}$ is a field tensor in $\Sigma^{(\mathrm{I})}$. The electric-field tensors can be defined in their principal axes by two parameters, namely the field-gradient anisotropy $\eta$ and the $Z^{*}$ component of the field gradient. Then the transformation of Eq. (4.1) can be expressed as

$$
\text { (1) } \begin{array}{r}
V_{-\zeta}{ }^{(2)}=* V_{0}^{(2)}\left[F_{-\zeta}(\alpha \beta \gamma, \Phi \Theta \Omega)\right. \\
\left.+\eta G_{-\zeta}(\alpha \beta \gamma, \Phi \Theta \Omega)\right],
\end{array}
$$

where the functions $F_{-\zeta}$ and $G_{-\zeta}$ can be calculated as a function of the Euler angles between these three sets of coordinate systems. With this field-gradient tensor operator, the electric quadrupole interaction Hamiltonian can be expressed as

$$
\begin{aligned}
V^{\mathrm{EQ}}=\sum_{\zeta}(-1)^{\zeta}\left[F_{\dashv \zeta}(\alpha \beta \gamma ; \Phi \Theta \Omega)+\right. & \left.\eta G_{-\zeta}(\alpha \beta \gamma ; \Phi \Theta \Omega)\right] \\
& { }^{*} V_{0}^{(2)} Q_{-\zeta}^{(2)},
\end{aligned}
$$

where ${ }^{*} V_{0}^{(2)}$ is an irreducible component of the field tensor in $\Sigma^{*}$.

By using the normal-mode expansion of the nuclear displacement as in the previous section, Eq. (4.3) can be expressed as

with

$$
V^{\mathrm{EQ}}\left(R_{1} R_{2}\right)=V_{0}^{\mathrm{EQ}}\left(R_{1} R_{2}\right)+V_{\tau}^{\mathrm{EQ}}\left(R_{2}\right),
$$

and

$$
V_{0}^{\mathrm{EQ}}\left(R_{1} R_{2}\right)=\sum_{\zeta}(-1)^{\zeta}\left[F_{-\zeta}(\alpha \beta \gamma ; \Phi \Theta \Omega)+\eta G_{-\zeta}(\alpha \beta \gamma, \Phi \Theta \Omega)\right]^{*} V_{0}^{(2)}(0) Q_{\zeta}{ }^{(2)},
$$

$$
\begin{array}{r}
V_{\tau}^{\mathrm{EQ}}\left(R_{2}\right)=\left(\frac{15 \hbar}{4 M_{c}}\right) \sum_{i}^{(n, n)} \sum_{\zeta} \sum_{\rho} \sum_{\mu \nu} \sum_{\mathbf{q}^{\prime} \mathbf{q}}(-1)^{\zeta} D_{\rho-\zeta^{(2)}}(\Phi \Theta \Omega)\left(\xi_{i} e / a_{0 c_{i}}{ }^{3}\right) \eta_{i \mu} \eta_{i \nu}\left[\omega(\mathbf{q} \mu) \omega\left(\mathbf{q}^{\prime} \nu\right)\right]^{-1 / 2}\left[d_{c i}-i \epsilon_{c i} q\left(\hat{q} \cdot \hat{a}_{0 c i}\right)\right] \\
\times\left[d_{c i}+i \epsilon_{c i} \mathbf{q}^{\prime}\left(\hat{q}^{\prime} \cdot \hat{a}_{0 c i}\right)\right] Y_{2}^{-\zeta}\left(\theta_{0 c i}, \varphi_{0 c i}\right) Q_{\zeta}\left({ }^{2}\right) a(\mathbf{q} \mu) a^{\dagger}\left(\mathbf{q}^{\prime} \nu\right) e^{i\left(\mathbf{q}-\mathbf{q}^{\prime}\right) \cdot \mathbf{x}_{0 c}},
\end{array}
$$

${ }^{19}$ F. Seitz and D. Turnbull, in Solid State Physics, edited by F. Seitz and D. Turnbull (Academic Press Inc., New York, 1957), Vol. 5 , p. 322

${ }_{20} \mathrm{~J}$. Van Kanen Donk, Physics 20, 781 (1954).

21 Von Hang Paul and Witlof Bummer, Ann. Physik 9, 323 (1962).

${ }^{22}$ K. Alder and R. M. Steffen, Phys. Rev. 129, 1199 (1963). 
where $R_{1}$ and $R_{2}$ represents the rotations of $R(\alpha \beta \gamma)$ and $R(\Phi \Theta \Omega)$. Equations (4.5) and (4.6) describes, respectively, the transformation of the static part and the dynamic part of the electric quadrupole coupling under the rotation of the crystal coordinate system. With this coupling Hamiltonian the function $\left\langle\frac{1}{2} \hbar \gamma_{\mathfrak{k}}\left(V_{\tau}{ }^{N c}\right)\right\rangle_{T}$, as given by Eq. (3.2), can be calculated as;

with

$$
\left\langle\frac{1}{2} \hbar \gamma_{\kappa}\left(V_{\tau}{ }^{\mathrm{EQ}}\left(R_{2}\right)\right)\right\rangle_{T}=\left\langle S_{\kappa}\left(V_{\tau}^{\mathrm{EQ}}\left(R_{2}\right)\right)\right\rangle_{T}-i\left\langle\frac{1}{2} \hbar \Gamma_{\kappa}\left(V_{\tau}^{\mathrm{EQ}}\left(R_{2}\right)\right)\right\rangle_{T},
$$

$$
\begin{aligned}
& \left\langle S_{\kappa}\left(V_{\tau} \mathrm{EQ}\left(R_{2}\right)\right)\right\rangle_{T}=\left(\frac{15 \hbar V}{4 M_{c} v^{3}}\right)^{2}\left|\left\langle I\left\|Q^{(2)}\right\| I\right\rangle\right|^{2} \sum_{\zeta=-2}^{2} \sum_{\alpha^{\prime} \neq k} \sum_{m m^{\prime}} \sum_{i}^{(n, n)} \sum_{\mu \nu}\left(\frac{\xi_{i} e}{a_{0 c i}{ }^{3}}\right)^{2}\left|F_{\zeta}\left(R_{2}, \theta_{0 c i} \varphi_{0 c i}\right)\right|^{2}\left(\begin{array}{ccc}
I & 2 & I \\
-m^{\prime} & \zeta & m
\end{array}\right)^{2} \\
& \times\left|\langle I m \mid \kappa\rangle\left\langle\kappa^{\prime} \mid \operatorname{Im} m^{\prime}\right\rangle\right|{ }^{2} \eta_{\imath \mu^{2}}{ }^{2} \eta_{i \nu}{ }^{2} P \int_{\omega_{\kappa \kappa^{\prime}}}^{\omega_{M}} d \omega \int_{0}^{\omega_{M-\omega_{\kappa \alpha^{\prime}}}} d \omega^{\prime} \omega \omega^{\prime} \int_{\Omega(\hat{q})} d \Omega \int_{\Omega^{\prime}\left(\hat{q}^{\prime}\right)} d \Omega^{\prime}\left[d_{c i}{ }^{2}+\epsilon_{c i}{ }^{2} \frac{\omega^{2}}{v^{2}}\left(\hat{q} \cdot \hat{a}_{0 c i}\right)^{2}\right] \\
& \times\left[d_{c i}{ }^{2}+\epsilon_{c 2}{ }^{{ }^{2}} \frac{v^{\prime 2}}{v^{2}}\left(\hat{q}^{\prime} \cdot \hat{a}_{0 c i}\right)^{2}\right] n_{T}(\omega)\left[n_{T}\left(\omega^{\prime}\right)+1\right]\left[\hbar \omega-\hbar \omega^{\prime}-k \omega_{\kappa k^{\prime}}\right]^{-1},
\end{aligned}
$$

and

$$
\begin{aligned}
& \left\langle\Gamma_{\kappa}\left(V_{\tau}^{\mathrm{EQ}}\left(R_{2}\right)\right)\right\rangle_{T}=2 \pi \hbar\left(15 V / 4 M_{c} v^{3}\right)^{2}\left|\left\langle I\left\|Q^{(2)}\right\| I\right\rangle\right|^{2} \sum_{\zeta=-2}^{2} \sum_{\kappa^{\prime} \neq \kappa} \sum_{m m^{\prime}} \sum_{i}^{(n, n)} \sum_{\mu \nu}\left(\frac{\xi_{i} e}{a_{0 c i}{ }^{3}}\right)^{2}\left|F_{\zeta}\left(R_{2} ; \theta_{0 c i} \varphi_{0 c i}\right)\right|^{2}\left(\begin{array}{ccc}
I & 2 & I \\
-m^{\prime} & \zeta & m
\end{array}\right)^{2}
\end{aligned}
$$

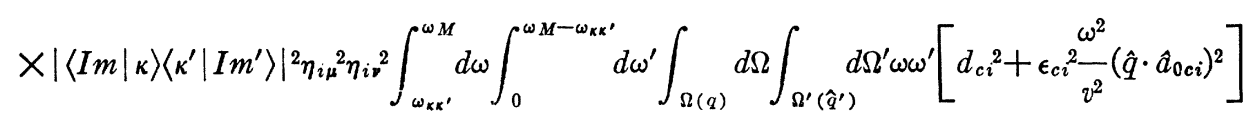

$$
\begin{aligned}
& \times\left[d_{c i}{ }^{2}+\epsilon_{c i}{ }^{\omega^{\prime 2}}{ }_{\vartheta^{2}}\left(\hat{q}^{\prime} \cdot \hat{a}_{0 c i}\right)^{2}\right] n_{T}(\omega)\left[n_{T}\left(\omega^{\prime}\right)+1\right] \delta\left(\hbar \omega-\hbar \omega^{\prime}-\hbar \omega_{\kappa k^{\prime}}\right),
\end{aligned}
$$

where

$$
F_{\zeta}\left(R_{2} ; \theta_{0 c i} \varphi_{0 c i}\right)=\sum_{\rho} D_{\rho \rightarrow \zeta}{ }^{(2)}(\Phi \Theta \Omega) Y_{2}^{-\zeta}\left(\theta_{0 c i} \varphi_{0 c i}\right) \text {. }
$$

The quantity $E_{\kappa}-E_{\kappa^{\prime}}$ in the denominator of Eq. (2.14) can be calculated as:

$$
\begin{aligned}
& E_{\kappa}\left(R_{1} R_{2}\right)-E_{\mathbf{k}^{\prime}}\left(R_{1} R_{2}\right)={ }^{*} V_{0}(2)(0)\left\langle I\left\|Q^{(2)}\right\| I\right\rangle \sum_{\zeta=-2}^{2} \sum_{m m^{\prime}}(-1)^{I-m^{\prime}}\left(\begin{array}{ccc}
I & 2 & I \\
-m^{\prime} & \zeta & m
\end{array}\right)\left[\left\langle I m^{\prime} \mid \kappa\right\rangle\langle\kappa \mid I m\rangle-\left\langle I m^{\prime} \mid \kappa^{\prime}\right\rangle\left\langle\kappa^{\prime} \mid I m\right\rangle\right] \\
& \times\left[F_{-\zeta}(\alpha \beta \gamma ; \Phi \Theta \Omega)+\eta G_{-\zeta}(\alpha \beta \gamma ; \Phi \Theta \Omega)\right] .
\end{aligned}
$$

where the irreducible component of the field tensor ${ }^{*} V_{0}{ }^{(2)}(0)$ is evaluated at the equilibrium positions of the lattice system in the principal axes.

In order to calculate the functions $\left\langle S_{x}\left(V_{\tau}{ }^{\mathrm{EQ}}\left(R_{2}\right)\right)\right\rangle_{T}$ and $\left\langle\frac{1}{2} \hbar \Gamma_{\kappa}\left(V_{\tau}{ }^{\mathrm{EQ}}\left(R_{2}\right)\right)\right\rangle_{T}$ one has to know the multiplication parameter $\xi_{i}$, the asymmetry of the crystalline field, the Euler angles between $\Sigma^{*}$ and $\Sigma^{(I I)}$, and the $z$ component of the field gradient. Once these data are available, one can find the reduced matrix element $\left\langle I\left\|Q^{(2)}\right\| I\right\rangle$ of the nuclear electric quadrupole moment in an excited state by comparing the experimentally measured anisotropy as a function of the rotations of the crystal axes with the theoretically calculated anisotropy.

\section{AN EXAMPLE FOR AN AXIALLY SYMMETRIC FIELD}

As an example of the application of the general theory, let us consider a simple case of a lattice system of octahedron structure. In a regular octahedron structure a nucleus will experience a cubic symmetric field. However, if we assume that an axial symmetric field exists due to a vacancy at a nearest neighboring lattice site, one immediately has the following properties; (1) $\eta=0$ for axial symmetry field, (2) the Euler angles between $\Sigma^{(I I)}$ and $\Sigma^{*}$ vanish, since the field is axially symmetric one can choose $X$ along $X^{*}$ and $Y$ along $Y^{*},(3) a_{0 c i}{ }^{*}=a$ the lattice constant, (4) $\rho_{1}=\cdots=\rho_{5}=\xi e, \rho_{6}=0$ due to a vacancy at the 6th neighbor, (5) $\epsilon_{c 1}=\cdots=\epsilon_{c 5}=1, \epsilon_{c 6}=0$ (here we have neglected the isotopic effect and assumed that all the nuclei have exactly the same mass so that $d_{c 1}=\cdots$ $\left.=d_{c 5}=0, d_{c 6}=1 / a\right)$, (6) $\eta_{i \mu} \eta_{i \nu}=\eta_{i \nu}{ }^{2} \delta_{\mu \nu}$ and all the angles of $\theta_{0 c i}$ and $\varphi_{0 c i}$ are known. Using these properties of the lattice system and making use of the fact that the phonons obey Bose statistics so that the number $n_{T}(\omega)$ of the phonons of energy $\hbar \omega$ present in a crystal at temperature $T$ is given by Planck's law $n_{T}(\omega)=\{\exp (\hbar \omega / k T)-1\}^{-1}$, (which we here approximate as $n_{T}(\omega) \simeq k T / \hbar \omega$ ), the perturbation factor for $180^{\circ}$ and $90^{\circ}$ correlation can now be 
calculated as:

$$
\begin{aligned}
& \bar{G}_{k_{1} k_{2}}{ }^{00}(\pi)=\sum_{m m^{\prime}} \sum_{\kappa k^{\prime}}(-1)^{2 I+m^{\prime}+m}\left[\left(2 k_{1}+1\right)\left(2 k_{2}+1\right)\right]^{1 / 2}\left(\begin{array}{ccc}
I & I & k_{1} \\
m^{\prime} & -m^{\prime} & 0
\end{array}\right)\left(\begin{array}{ccc}
I & I & k_{2} \\
m & -m & 0
\end{array}\right)\langle I m \mid \kappa\rangle\left\langle\kappa \mid I m^{\prime}\right\rangle \\
& X\left\langle I m^{\prime} \mid \kappa^{\prime}\right\rangle\left\langle\kappa^{\prime} \mid I m\right\rangle\left\{\left[1+B_{\kappa k^{\prime}}\left(R_{2}\right) T^{2}\right]-\left(i E_{\kappa k^{\prime}}\left(R_{1} R_{2}\right) \tau_{N} / \hbar\right)\left[1+G_{\kappa k^{\prime}}\left(R_{2}\right) T^{2}\right]\right\}^{-1}, \\
& \bar{G}_{k_{1} k_{2}}{ }^{0 N}(\pi / 2)=\sum_{m m^{\prime}} \sum_{\kappa k^{\prime}}(-1)^{2 I+m^{\prime}+m}\left[\left(2 k_{1}+1\right)\left(2 k_{2}+1\right)\right]^{1 / 2}\left(\begin{array}{ccc}
I & I & k_{1} \\
m^{\prime} & -m^{\prime} & 0
\end{array}\right)\left(\begin{array}{ccc}
I & I & k_{2} \\
m^{\prime \prime} & -m & N
\end{array}\right)\langle I m \mid \kappa\rangle\left\langle\kappa \mid I m^{\prime}\right\rangle \\
& \times\left\langle\operatorname{Im}^{\prime} \mid \kappa^{\prime}\right\rangle\left\langle\kappa^{\prime} \mid I m^{\prime \prime}\right\rangle\left\{\left[1+B_{\kappa \kappa^{\prime}}\left(R_{2}\right) T^{2}\right]-\left(i E_{\kappa k^{\prime}}\left(R_{1} R_{2}\right) \tau_{N} / \hbar\right)\left[1+G_{\kappa k^{\prime}}\left(R_{2}\right) T^{2}\right]\right\}^{-1},
\end{aligned}
$$

where

$$
\begin{aligned}
G_{\kappa \kappa^{\prime}}\left(R_{2}\right)=\left(\frac{15 k V \xi \xi \omega_{M^{2}}{ }^{2}}{8 \hbar M_{c} v^{5} a^{3}}\right)^{2}\left|\left\langle I\left\|Q^{(2)}\right\| I\right\rangle\right|^{2} \sum_{\zeta=-2}^{2} \sum_{m m^{\prime}} \sum_{i=1}^{5}\left(\begin{array}{ccc}
I & 2 & I \\
-m^{\prime} & \zeta & m
\end{array}\right)^{2}\left|F_{-\zeta}\left(\Theta_{z} ; \theta_{0 c i} \varphi_{0 c i}\right)\right|^{2}\left[\left\{\left(\hat{q} \cdot \hat{a}_{0 c i}\right)^{2}\right\}_{\Omega(\hat{q})}\right]^{2} \\
\\
\quad \times\left[\sum_{\alpha^{\prime \prime} \neq \kappa}\left|\langle I m \mid \kappa\rangle\left\langle\kappa^{\prime \prime} \mid I m^{\prime}\right\rangle\right|^{2}-\sum_{\kappa^{\prime \prime \prime} \neq \kappa^{\prime}}\left|\left\langle I m \mid \kappa^{\prime}\right\rangle\left\langle\kappa^{\prime \prime \prime} \mid I m^{\prime}\right\rangle\right|^{2}\right],
\end{aligned}
$$

and

$$
\begin{aligned}
B_{\kappa \kappa^{\prime}}\left(R_{2}\right)=\left(\pi \omega_{M} \tau_{N} / 5\right)\left(\frac{15 k V \xi e}{4 \hbar M_{c} v^{5} a^{3}}\right)^{2}\left|\left\langle I|| Q^{(2)} \| I\right\rangle^{2}\right| \sum_{\zeta=-2}^{2} \sum_{m m^{\prime}} \sum_{i=1}^{5}\left(\begin{array}{ccc}
I & 2 & I \\
-m^{\prime} & \zeta & m
\end{array}\right)^{2}\left[\left\{\left(\hat{q} \cdot \hat{a}_{0 c i}\right)^{2}\right\}_{\Omega(\hat{q})}\right]^{2} \\
\times\left|F_{\zeta}\left(\Theta_{z} ; \theta_{0 c i} \varphi_{0 c i}\right)\right|^{2}\left[\sum_{\kappa^{\prime \prime} \neq \kappa}\left|\langle I m \mid \kappa\rangle\left\langle\kappa^{\prime \prime} \mid \operatorname{Im}^{\prime}\right\rangle\right|^{2}+\sum_{\alpha^{\prime \prime \prime} \neq \kappa^{\prime}}\left|\left\langle I m \mid \kappa^{\prime}\right\rangle\left\langle\kappa^{\prime \prime \prime} \mid I m^{\prime}\right\rangle\right|^{2}\right] .
\end{aligned}
$$

We have kept the symmetry axis in the plane of the detectors and let $\Phi=\Omega=0$ in the rotation $R_{2}(\Phi \Theta \Omega)$. Then the perturbation factor depends on the angle $\Theta_{z}$ which is the orientation of symmetry axis with respect to the first detector. From Eqs. (2.12), (2.16), (5.1), and (5.2) one can calculate the rotational pattern of the anisotropy as a function of the orientation of the crystal axis. Clearly these equations are very complicated functions of $\Theta_{z}$ and no exact calculation has been attempted. However, if we make some simplification for illustrative purposes; namely by ignoring the rotational dependence of the unitary transformation coefficients and the level width due to the perturbation and evaluating the first derivatives of the function $G_{\kappa x^{\prime}}\left(\Theta_{z}\right)$ at zero rotation; then a considerably simplified solution for the condition $\partial A / \partial \Theta_{z}=0$ ( $A$ is the anisotropy function) can be calculated as;

$$
\left[\Theta_{z}\right]_{\max }=\frac{1}{2} \sin ^{-1}\left[\frac{\sum_{\kappa \kappa^{\prime}} G_{\kappa \kappa^{\prime}}(0) T^{2}}{\sum_{\kappa \kappa^{\prime}} A_{\kappa \kappa^{\prime}}}\right],
$$

where

$$
\begin{aligned}
A_{\kappa \kappa^{\prime}}={ }^{*} V_{0}^{(2)}(0)\left\langle I\left\|Q^{(2)}\right\| I\right\rangle \sum_{m m^{\prime}} \frac{(-1)^{I-m^{\prime}}}{2}\left[\left\langle I m^{\prime} \mid \kappa\right\rangle\langle\kappa \mid I m\rangle-\left\langle I m^{\prime} \mid \kappa^{\prime}\right\rangle\left\langle\kappa^{\prime} \mid I m\right\rangle\right] & \times\left[6^{1 / 2}\left(\begin{array}{ccc}
I & 2 & I \\
-m^{\prime} & 2 & m
\end{array}\right)-3\left(\begin{array}{ccc}
I & 2 & I \\
-m^{\prime} & 0 & m
\end{array}\right)\right]
\end{aligned}
$$

and

$$
\begin{aligned}
G_{\kappa \kappa^{\prime}}(0)=2\left(\frac{15 k V \xi e \omega_{M^{2}}}{8(h)^{1 / 2} M_{c} v^{5} a^{3}}\right)^{2}\left|\left\langle I\left\|Q^{(2)}\right\| I\right\rangle\right|^{2} \sum_{\zeta=-2}^{2} \sum_{m m^{\prime}} \sum_{i=1}^{5}\left(\begin{array}{ccc}
I & 2 & I \\
-m^{\prime} & \zeta & m
\end{array}\right)^{2} \omega_{\kappa \kappa^{\prime}} F_{\zeta}\left(0 ; \theta_{0 c 2} \varphi_{0 c i}\right) F_{\zeta}\left(0 ; \theta_{0 c i} \varphi_{0 c i}\right) \\
\times\left[\left\{\left(\hat{q} \cdot \hat{a}_{0 c i}\right)^{2}\right\rangle_{\Omega(\hat{q})}\right]^{2}\left[\sum_{\kappa^{\prime \prime \prime} \neq \kappa^{\prime}}\left|\left\langle I m \mid \kappa^{\prime}\right\rangle\left\langle\kappa^{\prime \prime \prime} \mid I m^{\prime}\right\rangle\right|^{2}-\sum_{\kappa^{\prime \prime} \neq \kappa}\left|\langle I m \mid \kappa\rangle\left\langle\kappa^{\prime \prime} \mid I m^{\prime}\right\rangle\right|^{2}\right] .
\end{aligned}
$$

The quantity $[\Theta]_{\max }$ is the angle between the symmetry axis and the first detector for the maximum anisotropy in the rotational pattern. From Eq. (5.5) one sees that when the crystal can be considered as a rigid lattice one always has $\left[\Theta_{z}\right]_{\max }=0$. This result has been pointed out by Abragam and Pound. ${ }^{14}$ However the present theory predicts for the rotational pattern a phase shift due to the thermal vibrations of the lattice system, and this simplified solution also predicts a $T^{2}$ dependence of the phase shift. The sign of the phase shift depends on the geometry of the experimental arrangement and the product of the reduced matrix element, $\left\langle I\left\|Q^{(2)}\right\| I\right\rangle$, of the nuclear electric quadrupole moment in the excited state and the $z$ component of the crystalline field gradient ${ }^{*} V_{0}^{(2)}(0)$. A phase shift in the rotational pattern of anisotropy 
was experimentally observed by Albers-Schönberg et $a .^{2}$ by using a metallic indium single crystal. Qualitatively, the observed phase shift can be explained from the present theory as due to the thermal vibrations of the lattice points. The effect of these thermal vibrations is to produce, at the site of the radioactive nucleus, an asymmetric fluctuating electric field gradient which is responsible for the phase shift in the rotational pattern of anisotropy.

\section{CONCLUSION}

In this formalism one has a systematic treatment which contains a description of the effects of the changes of the state of the environment. For a solid-state environment the present theory predicts a temperature dependence of the anisotropy.

The rotational dependence of the angular correlation has been developed for the cases of asymmetric crystalline fields. This will greatly increase the interpretability of experiments and the possibility of investigating nuclear quadrupole moments in excited states. For the case of an axially symmetric crystalline field, Eq. (5.5) predicts the phase shift in the rotational pattern of anisotropy as a function of crystal temperature.

The accuracy of the calculation of the nuclear electric quadrupole moment depends highly on the computations of the electrostatic field gradient at the nuclear site. The calculation of this crystalline field is very difficult and a model for the charge distribution in the lattice system is needed. Here we use the point-charge model and introduce an adjustable parameter $\xi_{i}$, to take the shielding, antishielding, and covalent effects into account. For the determination of this parameter one has to rely entirely on other measurements. ${ }^{20}$

Since a sufficient knowledge of the wave function of the electronic shell is available, ${ }^{23}$ the present theory [i.e., Eqs. (2.12) and (2.13)] will be suitable to investigate the effect of the nuclear spin relaxation due to coupling with atomic electrons on the angular correlation. ${ }^{24}$

${ }^{23}$ K. D. Bowers and J. Owen, Rept. Progr. Phys. 18, 304 (1955). ${ }^{24}$ H. J. Leisi and R. T. Deck, Phys. Rev. 129, 2117 (1962).

\title{
$\Lambda$-Binding Energies in Heavy Hyperfragments $(35 \leqslant A \leqslant 80)$
}

\author{
B. Bhowmik, T. Chand, D. V. Chopra, and D. P. Goyal \\ Department of Physics and Astrophysics, University of Delhi, Delhi, India \\ (Received 18 June 1965; revised manuscript received 12 November 1965)
}

\begin{abstract}
Analysis of 16 short-range $(\leqslant 10 \mu)$ hyperfragments, produced from $1.5-\mathrm{GeV} / c K^{-}$interactions in $L_{4}$ hypersensitized emulsions and decaying $\pi^{-}$-mesonically, is presented. Of these, 9 are classified as due to the residual spallation products of silver and bromine nuclei, 5 as beloning to lighter species $(A \leqslant 20)$, and 2 as ambiguous. The masses of the 9 spallation hyperfragments are determined using the "spallation model." It is concluded that for hyperfragments of masses $A \simeq 35$ to 80 , the upper limits of $B_{\Lambda}$ could vary from 21.9 to $24.5 \mathrm{MeV}$.
\end{abstract}

\section{INTRODUCTION}

$\mathbf{F}$ OLLOWING Jones et al., ${ }^{1}$ it is now generally believed that the majority of hyperfragments (HF's) of ranges $\leqslant 10 \mu$ produced by high-energy $K^{-}$interactions at momenta $0.8,{ }^{1-3} 1.3$ and $1.5,{ }^{4} 2.2,{ }^{5}$ and $3.0^{6}$

\footnotetext{
${ }^{1}$ B. D. Jones, B. Sanjeevaiah, J. Zakrzewski, M. CsejtheyBarth, J. P. Lagnaux, J. Sacton, M. J. Beniston, E. H. S. Burhop, and D. H. Davis, Phys. Rev. 127, 236 (1962).

2 N. A. Nickols, S. B. Curtis, and D. J. Prowse, Phys. Letters 1 327 (1962).

${ }^{3}$ I. R. Kenyon, Nuovo Cimento 29, 589 (1963).

${ }^{4}$ E. R. Fletcher, J. Lemonne, P. Renard, J. Sacton, D. O'Sullivan, T. P. Shah, A. Thompson, P. Allen, Sr., M. Heeran, A. Montwill, J. E. Allen, M. J. Beniston, D. A. Garbutt, R. C. Kumar, P. V. March, T. Pniewski, and J. Zakrzewski, Phys. Letters 3, 280 (1963).

${ }^{5}$ R. J. Prem and P. H. Steinberg, University of Maryland report, 1963 (unpublished).

${ }^{6}$ J. Lemonne, J. Sacton, G. Schorochoff, M. A. Shaukat, W. T. Toner, P. Allen, D. H. Davis, E. R. Fletcher, J. E. Allen, V. A. Bull, M. M. Kasim, P. V. March, K. Pniewska, T. Pniewski, M. Suk, M. Votruba, J. Herynek, J. Piekarz, and J. Zakrzewski, Université de Bruxelles-Bulletin No. 18, 1964 (unpublished).
}

$\mathrm{GeV} / \mathrm{c}$ and of ranges $\leqslant 3 \mu$ produced by $K^{-}$-at-rest ${ }^{7}$ interactions are the residual spallation products of silver and bromine nuclei, and possess mass numbers in the range $A \simeq 20$ to 100 , the limits on $A$ depending on the $K^{-}$momentum used. The determination of the $\Lambda$ binding energies $\left(B_{\Lambda}\right)$ and masses of these heavy8 HF's is of great interest since these can be effectively utilized for estimating the potential well-depth $D_{\Lambda}$ seen by a $\Lambda$ particle in nuclear matter.

In their first investigation, Davis et al..$^{9}$ used $K^{-}$ interactions at momenta $0,0.8$, and $1.5 \mathrm{GeV} / c$ and reported 5 examples of $\pi^{-}$-mesonic decays of short-range HF's attributed to mass numbers $A \simeq 60$ to 100 . Although the calculated values of the upper limits of

7 J. Lemonne, C. Mayeur, J. Sacton, D. H. Davis, D. A Garbutt, and J. E. Allen, Nuovo Cimento 34, 529 (1964).

${ }^{8}$ Henceforth we shall denote HF's having $A>20$ as "heavy," $A \leqslant 20$ as "light."

${ }^{9}$ D. H. Davis, R. Levi Setti, M. Raymund, O. Skjeggestad, G. Tomasini, J. Lemonne, P. Renard, and J. Sacton, Phys. Rev. Letters 9, 464 (1962). 